\title{
A Nationwide Seroepidemiologic Study on $Q$ Fever Antibodies in Sheep of Portugal
}

\author{
Rita Cruz, Fernando Esteves, Carmen Vasconcelos-Nóbrega, Carla Santos, \\ Ana S. Ferreira, Cristina Mega, Ana C. Coelho, Helena Vala,,4 and João R. Mesquita, ${ }^{1,5}$
}

\begin{abstract}
Introduction: Q fever is an almost global zoonotic disease caused by Coxiella burnetii. Human infections can produce acute and chronic disease that can lead to abortions and stillbirths in pregnant women, usually infected by the inhalation of $C$. burnetii-contaminated aerosols or through consumption of contaminated products. Sheep are one of the primary animal reservoirs with disease being associated with vast shedding of bacteria in placentas, feces, milk, and birth fluids. Although almost neglected in the past, recent outbreaks of sheep origin have alerted the public and the scientific community.

Materials and Methods: An epidemiologic survey to estimate the seroprevalence of Q fever antibodies was performed in a representative number of sheep of all regions of continental Portugal $(n=1068)$, using a commercial ELISA (ID Screen Q Fever Indirect Multi-species Kit; IDvet ${ }^{\mathrm{TM}}$, Montpellier, France).

Results and Discussion: An anti-C. burnetii seroprevalence of $11.4 \%$ (95\% confidence interval 9.6-13.5) was found, with a clear distinction between the Center region with highest seroprevalence, and the rest of the territory. Sheep traditional farming is widely present in Portugal and is part of the cultural and gastronomical background of the country. This close proximity to small ruminants may contribute to the zoonotic transfer to humans.
\end{abstract}

Keywords: ELISA, Portugal, Q fever, seroprevalence, sheep

\section{Introduction}

Q FEVER IS AN ALMOST GLOBAL zoonotic disease caused by Coxiella burnetii, which is able to infect several animal species and of which cattle, sheep and goats are the primary animal reservoirs (Van den Brom et al. 2015, Khor et al. 2018). Q fever in humans can produce acute disease that usually leads to pneumonia, hepatitis, and self-limited illness (Guatteo et al. 2011). Chronicity is associated to endocarditis in immuno-compromised individuals or abortions and stillbirths in pregnant women (Angelakis and Raoult 2010, Dabaja et al. 2018). Human infection occurs by C. burnetiicontaminated aerosols of animal origin that are inhaled (Guatteo et al. 2011). Although rare, oral transmission by consumption of contaminated dairy products is also possible as is also sexual and vertical transmission (Kruszewska and Tylewska-Wierzbanowska 1997, Milazzo et al. 2001). Though almost neglected in the past, recent outbreaks have alerted the public and the scientific community (van der Hoek et al. 2010). In The Netherlands, a large outbreak affecting 2357 human cases that lasted several years was notified in 2009 and linked to abortion waves on dairy goat flocks (van der Hoek et al. 2010). In fact, $\mathrm{Q}$ fever in small ruminants (sheep and goats) is associated with vast shedding of bacteria in placentas, feces, milk, and birth fluids, and usually manifest as abortions, which greatly increases the risk of disease spread (O'Neill et al. 2014, Filioussis et al. 2017).

In the Iberic Peninsula, very few regional studies on $\mathrm{Q}$ fever in ruminants have been made (Anastácio et al. 2013, Cumbassá et al. 2015), and no serologic survey in the full continental

\footnotetext{
${ }^{1}$ Centre for Studies in Education and Health Technologies (CI\&DETS), Agrarian School of Viseu, Polytechnic Institute of Viseu, Viseu, Portugal.

${ }^{2}$ Laboratory of Microbiology, Department of Biological Sciences, Faculty of Pharmacy, University of Oporto, Porto, Portugal.

${ }^{3}$ Animal and Veterinary Research Centre (CECAV), University of Trás-os-Montes and Alto Douro, Vila Real, Portugal.

${ }^{4}$ Centre for the Research and Technology of Agro-Environmental and Biological Sciences (CITAB), University of Trás-os-Montes and Alto Douro, Vila Real, Portugal.

${ }^{5}$ Epidemiology Research Unit (EPIUnit), Institute of Public Health, University of Porto, Porto, Portugal.
} 
territory Portugal has ever been done. Hence, an epidemiologic survey was set up to estimate the seroprevalence of Q fever antibodies in sheep of all regions of Portugal.

\section{Materials and Methods}

\section{Sample size}

This study used samples collected in 2014 for a previous study (Esteves et al. 2016). Sample size was calculated considering the following a priori assumptions: population size of 2,092,175 sheep (IFAP 2018), an expected C. burnetii seroprevalence of $50 \%$ (allowing for the largest sample possible), an absolute error of $3 \%$, and a $95 \%$ confidence level (DESCRIBE package, WINPEPI updated, version 11.43). A calculated sample size of 1068 sheep was obtained and a stratified random sampling design was performed by categorizing according to the Nomenclature of Territorial Units for Statistics level II (NUTS II) regions (North, Center, Lisboa and Vale do Tejo, Alentejo, and Algarve), to reduce possible confounders associated to the heterogeneous geographical distribution of sheep in Portugal. The 2014 official animal census data of Portugal reported the following distribution on sheep head according to region: 315,506 sheep are located in the North $(15.1 \%), 481,017$ in the Center $(23 \%), 42,861$ in Lisboa and Vale do Tejo (2\%), 1,206,876 in Alentejo (57.7\%), and 45,915 in Algarve (2.2\%) (IFAP 2018). As no herd level statistics are available, to better represent the distribution in the five regions of continental Portugal, samples from four farms spread within each region (at the North, South, East, and West) were selected for screening.

\section{ELISA screening}

Blood samples had been collected aseptically by jugular vein puncture into sterile labeled Vacutainer tubes without additives (BD Vacutainer Systems, Plymouth, United Kingdom). Samples were kept cold during transport to the laboratory. Sera was removed after centrifugation and stored at $-20^{\circ} \mathrm{C}$ until analysis. All sera were from healthy female sheep with 6 months to 10 years of age (average age of 5 years) born in Portugal, randomly selected upon the moment of the official brucellosis control. In total, 161 samples from the North, 246 from the Center, 21 from Lisboa and Vale do Tejo, 616 from Alentejo, and 24 from Algarve were selected. Sera were tested for the presence of anti-C. burnetii IgG antibodies using a commercial indirect ELISA, ID Screen Q Fever Indirect
Multi-species Kit (IDvet ${ }^{\mathrm{TM}}$, Montpellier, France), following the manufacturer's instructions. Sensitivity and specificity of this assay has shown to be $100 \%$ (IDvet, according to the manufacturer internal validation report). Briefly, sample-topositive control $(\mathrm{S} / \mathrm{P})$ ratio in each serum was calculated according to the formula provided: $\mathrm{S} / \mathrm{P}=\left(\mathrm{OD}_{450}\right.$ sample $-\mathrm{OD}_{450}$ $\mathrm{NC}) /\left(\mathrm{OD}_{450} \mathrm{PC}-\mathrm{OD}_{450} \mathrm{NC}\right)$; where $\mathrm{OD}_{450}$ sample $=$ optical density of the sample, $\mathrm{OD}_{450} \mathrm{NC}=$ optical density of the negative control, and $\mathrm{OD}_{450} \mathrm{PC}=$ optical density of the positive control. Results were expressed as an index $(\mathrm{S} / \mathrm{P} \times 100)$. Indices stratified as four different rising categories. Samples with S/P indices $<40 \%$ were considered negative, samples with $\mathrm{S} / \mathrm{P}$ indices between $40 \%$ and $50 \%$ were considered doubtful, samples with S/P indices between $50 \%$ and $80 \%$ were considered low positive, and samples with $\mathrm{S} / \mathrm{P}$ indices $>80 \%$ were considered strong positive. Doubtful samples were retested and if resulting doubtful, considered as negative. Obtained data were used to calculate NUTS II-specific seroprevalence values. Exact binomial 95\% confidence intervals (CI) were established for proportions.

\section{Results and Discussion}

The presence of anti-C. burnetii antibodies was found in 122 sheep, which represents a $11.4 \%$ (95\% CI 9.6-13.5) seroprevalence of IgG anti-C. burnetii in sheep of Portugal. Of these 122 positive sheep, 53 (43.4\%, 95\% CI 34.5-52.7) were considered low positive and 69 (56.6\%, 95\% CI 47.365.5) considered strong positive. Regarding the distributions according to regions, anti-C. burnetii were found in 18 of 161 sheep of the North of Portugal (11.2\%, 95\% CI 6.8-17.1), of which 7 (38.9\%; 95\% CI 17.3-64.3) were low positive and 11 $(61.1 \%, 95 \%$ CI 35.7-82.7) were strong positive; in 44 of 246 sheep of the Center of Portugal (17.9\%, 95\% CI 13.3-23.3), $13(29.5 \%, 95 \%$ CI 16.8-45.2) were low positive and 31 (70.5\%, 95\% CI 54.8-83.2) were strong positive; in none of the 21 sheep from Lisboa and Vale do Tejo $(0 \%, 95 \%$ CI 0.0 $0.0)$; in 59 of the 616 sheep of Alentejo $(9.6 \%, 95 \%$ CI 7.4 $11.9), 32(54.2 \%, 95 \%$ CI 40.8-67.3) were low positive and 27 (45.8\%, 95\% CI 32.7-59.2) were strong positive; and only 1 of the 24 sheep of Algarve (4.2\%, 95\% CI 0.1-21.1) was considered low positive (100\%, 95\% CI 2.5-100) (Table 1; Fig. 1). The seroprevalence found in Portugal, even considering individual regions, appears to be within the low ranges when comparing with the seroprevalence observed among sheep in the neighboring country of Spain, reported to be

Table 1. Screening for Anti-Coxiella burnetit IgG Antibodies in 1068 Sheep of All Five Regions of Continental Portugal

\begin{tabular}{lccc}
\hline & $\begin{array}{c}\text { Anti-C. burnetii low pos/ } \\
\text { region total pos: } \text { no. }(\% ; C I)\end{array}$ & $\begin{array}{c}\text { Anti-C. burnetii strong pos/ } \\
\text { region total pos: } \text { no. }(\% ; \text { CI) }\end{array}$ & $\begin{array}{c}\text { Anti-C. burnetii pos/ } \\
\text { total: } \text { no. }(\% ; \text { CI) }\end{array}$ \\
\hline Region & & & \\
$\quad$ North & $7 / 18(38.9 ; 17.3-64.3)$ & $11 / 18(61.1 ; 35.7-82.7)$ & $18 / 161(11.2 ; 6.8-17.1)$ \\
Center & $13 / 44(29.6 ; 16.8-45.2)$ & $31 / 44(70.4 ; 54.8-83.2)$ & $44 / 246(17.9 ; 13.3-23.3)$ \\
Lisboa and & $0 / 0(0.0 ; 0.0-0.0)$ & $0 / 0(0.0 ; 0.0-0.0)$ & $0 / 21(0.0 ; 0.0-0.0)$ \\
$\quad$ Vale do Tejo & $32 / 59(54.2 ; 40.8-67.3)$ & $27 / 59(45.8 ; 32.7-59.2)$ & $59 / 616(9.6 ; 7.4-11.9)$ \\
Alentejo & $1 / 1(100 ; 2.5-100)$ & $0 / 1(0 ; 0-97.5)$ & $1 / 24(4.2 ; 0.1-21.1)$ \\
Algarve & $53 / 122(43.4 ; 34.5-52.7)$ & $69 / 122(56.6 ; 47.3-65.5)$ & $122 / 1068(11.4 ; 9.6-13.5)$ \\
Total & & &
\end{tabular}

CI, $95 \%$ confidence interval. 


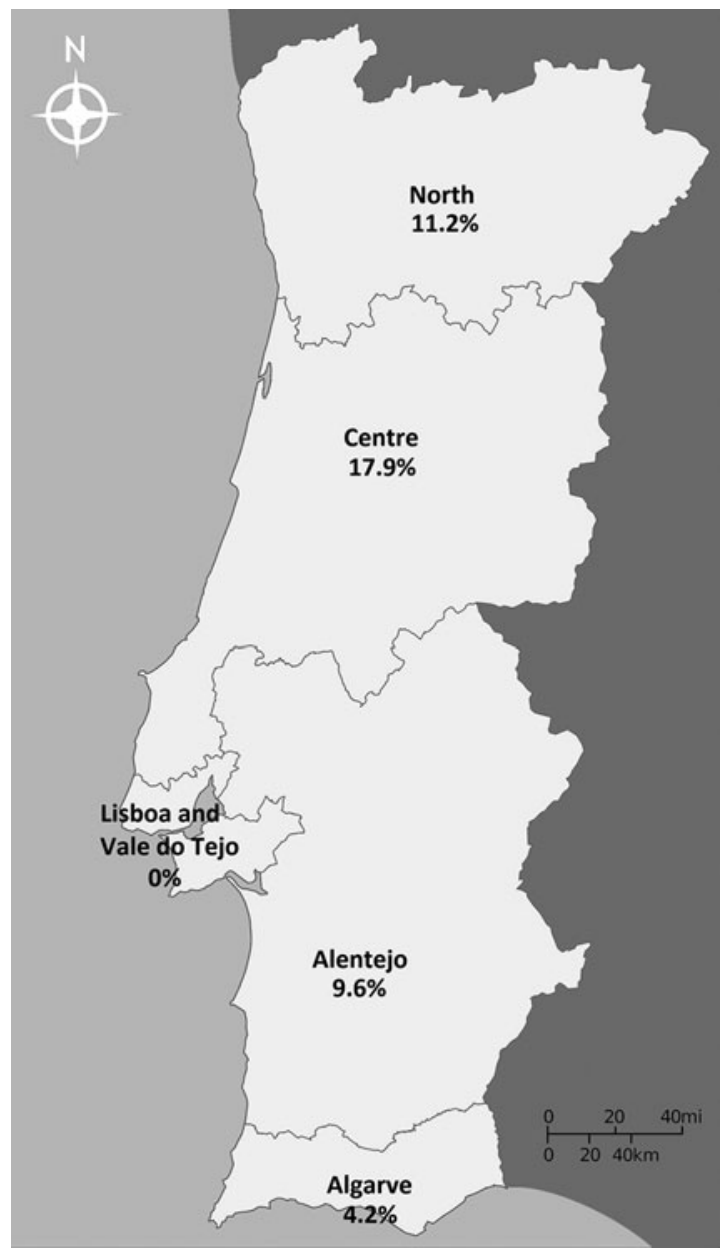

FIG. 1. Percentages of anti-Coxiella burnetii seropositive sheep in the five NUTS II regions of continental Portugal.

from $9.8 \%$ in the Basque country (northern country) to $\sim 30 \%$ in the islands of Canarias, considered a highly endemic region (García-Pérez et al. 2009, Rodriguez et al. 2010, Ruiz-Fons et al. 2010, Fernández-Aguilar et al. 2016, Bolaños-Rivero et al. 2017). In fact seroprevalence in sheep is estimated to be around 15-20\% in many countries of the world (Guatteo et al. 2011), which confirms that sheep of Portugal showed relatively low $\mathrm{Q}$ fever seroprevalence.

When comparing anti-C. burnetii presence in sheep according to NUTS II distribution, a higher seroprevalence in the Center region can be observed when comparing to the other regions of Portugal. Unlike the regions south of the Center region (Alentejo, Lisboa, and Vale do Tejo and Algarve) that have vast flat plains and low density extensive husbandry systems, the North region has a highly irregular mountainous terrain (favoring intensive husbandry) and the Center has a mixture of hills and mountains that transition to plains to the south (Tibério and Dinis 2014). This terrain profile shifts from mountainous to plains in the Center region and favors semiextensive husbandry systems, where sheep are housed during the night and are allowed movement during the day (Fraga et al. 2014, Tibério and Dinis 2014). The semi-extensive husbandry that allows use of common pasture by sheep during the day can favor infection by spore-like forms that are deposited in the soil and have a long survival time, helping ex- plain the increased anti-C. burnetii seroprevalence in this region. Also from a climatic standpoint the country presents a relatively large set of mesoclimates, spanning from dryer in the Southern regions, to more humid and windy in the North and Center regions (Santos et al. 2012). This windy climate in the Center region may also favor $C$. burnetii airborne dispersion and transmission in the outdoor environment and help explain the increased seroprevalence in this region, when compared to the remaining regions of Portugal.

To this moment only one sheep seroprevalence study has been performed in Portugal, collecting blood from 2011 (Anastácio et al. 2013). In that study, the global individual seroprevalence was $8.6 \%$ and animals belonged to the Center region of Portugal, which is lower than the seroprevalence detected in this study for that region and may indicate an increasing circulation of $C$. burnetii. However, caution must be taken in this comparison since different enzyme immunoassays were used along with different sampling designs.

In conclusion, this is the first nationwide seroepidemiologic study that surveyed Q fever in sheep in Portugal also profiling the distribution of seropositive animals according to regions. Although seroprevalence seems low, the agent appears to be distributed across the country and hence alerts for the possibility of zoonotic transmission have to be made. Moreover, sheep traditional farming is widely present in Portugal and is part of the cultural and gastronomical background of the country. This close proximity to small ruminants may contribute to the zoonotic transfer to humans. Although preliminary results from this study show relatively low Q fever seroprevalence in Portugal, there is the need to provide a clearer understanding of $C$. burnetii epidemiology in Portugal. Implementing monitoring programs on sentinel herds may help prevent or mitigate the effects of potential epidemics.

\section{Acknowledgments}

This work is financed by national funds through FCTFundação para a Ciência e Tecnologia, I.P., under the projects UID/Multi/04016/2016, FCOMP-01-0124-FEDER-009525, UID/AGR/04033/2013, and SBVEPS (Proc ${ }^{\circ} 441.00$ SERVIA); QREN/FEDER under the project Ovislab ICT-201305-004-5314 ID-64757; CI\&DETS and CGD under projects rumDISEASE PROJ/CI\&DETS/2016/0023, SBMERGE PROJ/CI\&DETS/CGD/009, and HEALTHY-ValorWhey PROJ/CI\&DETS/CGD/007); and FEDER/COMPETE/POCI under project POCI-01-0145-FEDER-006958. We would like to thank to UTAD and CITAB for their support.

\section{Author Disclosure Statement}

No conflicting financial interests exist.

\section{References}

Anastácio S, Tavares N, Carolino N, Sidi-Boumedine K, et al. Serological evidence of exposure to Coxiella burnetii in sheep and goats in central Portugal. Vet Microbiol 2013; 167: 500-505.

Angelakis E, Raoult D. Q Fever. Vet Microbiol 2010; 140: 297-309.

Bolaños-Rivero M, Carranza-Rodríguez C, Rodríguez NF, Gutiérrez C, et al. Detection of Coxiella burnetii DNA in peridomestic and wild animals and ticks in an endemic region 
(Canary Islands, Spain). Vector Borne Zoonotic Dis 2017; 17: 630-634.

Cumbassá A, Barahona MJ, Cunha MV, Azórin B, et al. Coxiella burnetii DNA detected in domestic ruminants and wildlife from Portugal. Vet Microbiol 2015; 180:136-141.

Dabaja MF, Greco G, Villari S, Bayan A, et al. The First Serological Study of Q fever in humans in Lebanon. Vector Borne Zoonotic Dis 2018; 18:138-143.

Esteves F, Mesquita JR, Vala H, Abreu-Silva J, et al. Serological evidence for Schmallenberg virus infection in Sheep of Portugal, 2014. Vector Borne Zoonotic Dis 2016; 16:63-65.

Fernández-Aguilar X, Cabezón Ó, Colom-Cadena A, Lavín S, et al. Serological survey of Coxiella burnetii at the wildlifelivestock interface in the Eastern Pyrenees, Spain. Acta Vet Scand 2016; 58:26.

Filioussis G, Theodoridis A, Papadopoulos D, Gelasakis AI, et al. Serological prevalence of Coxiella burnetii in dairy goats and ewes diagnosed with adverse pregnancy outcomes in Greece. Ann Agric Environ Med 2017; 24:702-705.

Fraga H, Malheiro AC, Moutinho-Pereira J, Cardoso RM, et al. Integrated analysis of climate, soil, topography and vegetative growth in Iberian viticultural regions. PLoS One 2014; 9: e108078.

García-Pérez AL, Astobiza I, Barandika JF, Atxaerandio R, et al. Short communication: Investigation of Coxiella burnetii occurrence in dairy sheep flocks by bulk-tank milk analysis and antibody level determination. J Dairy Sci 2009; 92:1581-1584.

Guatteo R, Seegers H, Taurel AF, Joly A, et al. Prevalence of Coxiella burnetii infection in domestic ruminants: A critical review. Vet Microbiol 2011; 149:1-16.

IFAP. Instituto Financiamento da Agricultura e Pescas. 2018. Available at www.ifap.min-agricultura.pt/portal/page/portal/ F73E9028F8EB5096E043C0A8C8C55096

Khor CS, Mohd-Rahim NF, Hassan H, Chandren JR, et al. Seroprevalence of $\mathrm{Q}$ fever among the indigenous people
(Orang Asli) of Peninsular Malaysia. Vector Borne Zoonotic Dis 2018; 18:131-137.

Kruszewska D, Tylewska-Wierzbanowska S. Isolation of Coxiella burnetii from bull semen. Res Vet Sci 1997; 62:299-300.

Milazzo A, Hall R, Storm PA, Harris RJ, et al. Sexually transmitted Q fever. Clin Infect Dis 2001; 33:399-402.

O’Neill TJ, Sargeant JM, Poljak Z. A systematic review and meta-analysis of phase I inactivated vaccines to reduce shedding of Coxiella burnetii from sheep and goats from routes of public health importance. Zoonoses Public Health 2014; 61: 519-533.

Ruiz-Fons F, Astobiza I, Barandika JF, Hurtado A, et al. Seroepidemiological study of $\mathrm{Q}$ fever in domestic ruminants in semi-extensive grazing systems. BMC Vet Res 2010; 6:3.

Santos JA, Malheiro AC, Pinto JG, Jones GV. Macroclimate and viticultural zoning in Europe: Observed trends and atmospheric forcing. Clim Res 2012:89-103.

Tibério ML, Diniz F. Sheep and goat production in Portugal: A dynamic view. Modern Econ 2014; 5:6.

Van den Brom R, van Engelen E, Roest HI, van der Hoek W, et al. Coxiella burnetii infections in sheep or goats: An opinionated review. Vet Microbiol 2015; 181:119-129.

van der Hoek W, Dijkstra F, Schimmer B, Schneeberger PM, et al. Q fever in the Netherlands: An update on the epidemiology and control measures. Euro Surveill 2010; 15pii:19520.

Address correspondence to: João R. Mesquita Escola Superior Agrária de Viseu Quinta da Alagoa

Estrada de Nelas, Ranhados Viseu 3500-606 Portugal

E-mail: jmesquita@esav.ipv.pt 